\title{
Tension pneumocephalus attributable to an ethmoid osteoma presenting as a stroke in evolution: an unusual presentation
}

\author{
D C Bramley, S Ghosh
}

A 63 year old white man presented to the accident and emergency department with a 24 hour history of gradual onset of mild weakness of his left upper limb, which progressed to involve his left lower limb. There was no history of any other CNS symptoms. He also stated that the paresis varied with posturebeing worse on standing and sitting, while it was relieved by lying down; it was also associated with a noticeably diminished shortterm memory over the past few weeks. Clinical examination confirmed a mild left hemiparesis (grade 4/5), with brisk reflexes and an "equivocal" plantar response on the left. The rest of the examination was unremarkable: he was normotensive, no carotid bruits were auscultated and the fundi were normal. Haematological and biochemical investigations were normal. A diagnosis of a progressive cerebrovascular accident (CVA) was made and he was admitted to hospital for further observation.

Over the next few days, his hemiparesis slightly worsened, and routine computed tomography was carried out four days later. Routine axial sections (fig 1) revealed a $5 \mathrm{~cm}$ diameter pneumocephalus, and a mass with irregular calcification was noted arising from the base of skull in the right ethmoid sinuses (fig 2), encroaching on to the orbit. He was transferred to the regional ENT/neurosurgical centre, where a surgical intervention was decided upon. He underwent a simultaneous lateral rhinotomy and bilateral frontal craniotomy with an osteoplastic flap. A complete excision of his osteoma was performed, apart from a small area near the orbital apex. Some $40 \mathrm{ml}$ of air were found trapped intracranially, and the osteoma was associated with multiple mucoceles, which was responsible for the irregular appearance on computed tomography. His skull base defect was repaired with a fascia lata graft from his thigh. He made an uneventful recovery and his paresis improved immediately. His only persistent neurological deficit is anosmia. Subsequent histopathological examination confirmed the diagnosis of an osteoma, with dense trabeculae and little evidence of osteoblastic activity.

Primary osteomas of the ethmoid sinuses Dr Bramley, 108 Burgoyne Road, Sheffield S6 3OB, UK (D.C.Bramley@doctors.org.uk)

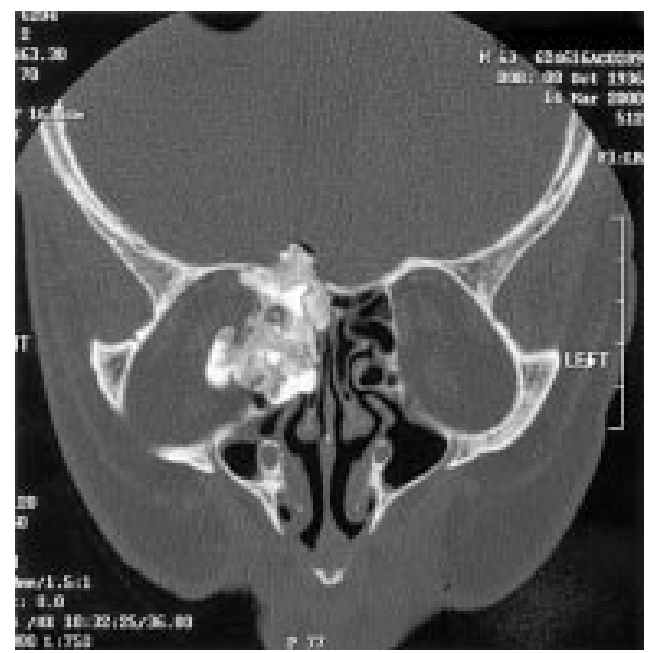

Figure 1 Coronal CT scan showing the osteoma with associated mucocele.

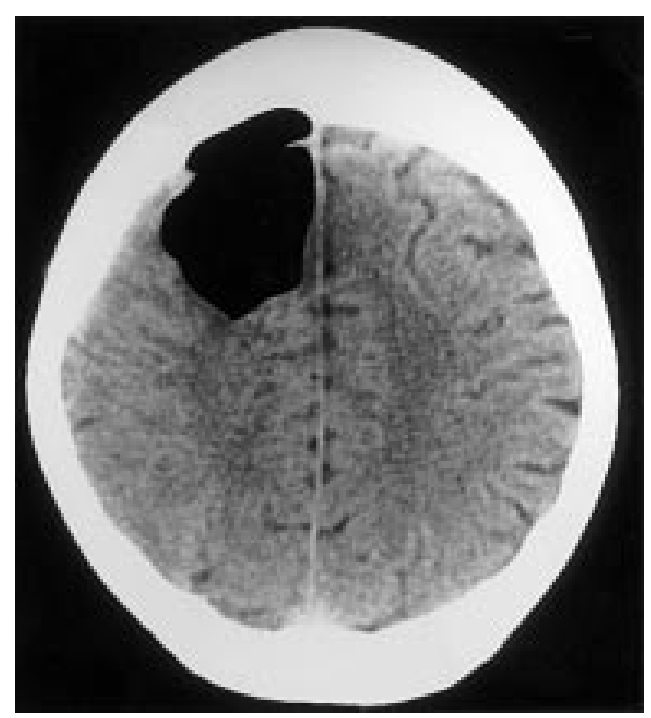

Figure 2 Axial CT scan showing the frontal location of the pneumocephalus. Patient supine.

cephalus is an unusual complication. They have been known to present acutely with fits, headaches, dementia, ${ }^{1}$ cerebral abscesses,${ }^{2}$ and acute cerebral hypertension. ${ }^{3}$ In this case the striking postural hemiparesis was presumably 
attributable to the tension pneumocephalus compressing the cerebral cortex. Interestingly, it is probable that the pneumocephalus would have been apparent from initial presentation on a plain skull radiograph.

In conclusion, spontaneous pneumocephalus is one of the rare conditions that should be considered in the differential diagnosis of raised intracranial pressure and atypical neurological symptoms including "change with posture".

\section{Contributors}

$\mathrm{Mr}$ A J Parker was responsible for the diagnosis and management of this patient, and provided information regard- ing this case, and also the more usual presentations of osteomas. Mr A Panarese participated in the background research involved in this paper and with editing the paper.

Funding: none.

Conflicts of interest: none.

1 George J, Merry GS, Jellet LB, et al. Frontal sinus osteoma with complicating intracranial aerocoele. Aust $N Z \mathcal{F}$ Surg with complica

2 Holness RO, Attia E. Osteoma of the fronto-ethmoidal sinus with secondary brain abscess and intracranial mucocele: case report. [Letter]. Neurosurgery 1995;36:1237-38.

3 Pennau D, Dubin J, Faivre J, et al. Acute intracranial hypertension. Pneumatocoele-osteoma of the frontal sinus. [In French]. Revue d'Oto-Neur-Opthalmologie 1976;48:57-70.

\section{Hallucal sesamoid bone stress fracture; 21 st century "club foot"}

\section{R Singh, N Slater}

\section{Case report}

A 24 year old woman complained of pain and swelling plantar surface of her right great toe after spending many hours dancing in a night club. This was her principal social activity. Although tall, she wore high heels. Examination revealed tenderness localised to the fibular sesamoid and radiography (fig 1) confirmed a longitudinal fracture of this bone. Symptoms settled rapidly with conservative treatment including use of flat soled trainers.

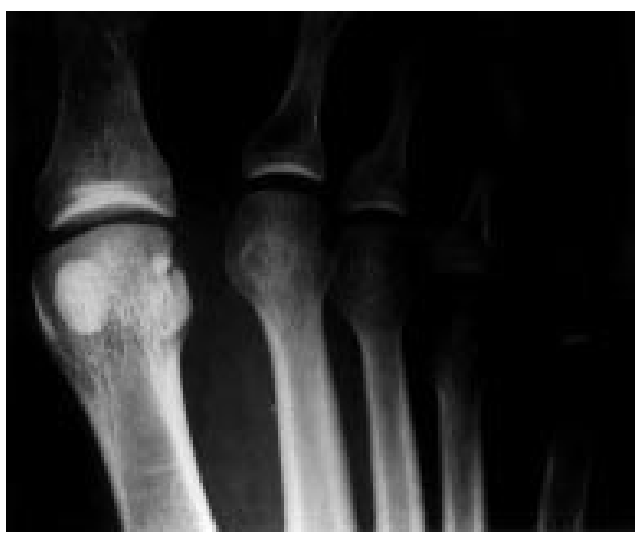

Figure 1 Anteroposterior non-weight bearing radiograph of the right forefoot showing a recent longitudinal fracture of the fibular sesamoid.

\section{Discussion}

Tibial and fibular sesamoid bones occur within the tendon slips of flexor hallucis brevis beneath the first metatarsal head; they increase the mechanical advantage of this muscle during the 'toe-off' phase of locomotion and may also share weight bearing. ${ }^{1}$ Fractures and sesamoiditis, most commonly of the tibial sesamoid, are seen in professional dancers ${ }^{2}$ and athletes, ${ }^{3}$ probably caused by compressive forces between sesamoid and metatarsal head but possibly by repeated tensile forces from vigorous "toe-off" activity.

High heels flex the first metatarsophalangeal joint and increase compressive forces on the sesamoids. This, and many hours of vigorous dancing probably combined to cause the injury reported here. Widespread use of recreational drugs that enhance mood and energy and might persuade a person to ignore skeletal discomfort may also contribute to this "club foot". Clubbing culture shows no signs of abating and we believe this injury will be recognised increasingly in future with the accident and emergency department as probable first site of presentation.

\footnotetext{
1 Yamaguchi Y. Biomechanical investigation of the sesamoid bones of the hallux. Fournal of the fapanese Orthopaedic Association 1993;67:211-20.

2 Quirk R. Common foot and ankle injuries in dance. Orthop Clin North Am 1994;25:123-33.

3 Richardson G. Injuries to the hallucal sesamoids in the athlete. Foot Ankle 1987;7:234.
} 


\title{
Tension pneumothorax: a difficult diagnosis
}

\author{
B L Watts, M A Howell
}

A 65 year old man was brought into the resuscitation room in cardiac arrest. Forty five minutes earlier he had become short of breath before collapsing. The only other history available was that he had undergone a left pneumonectomy several years previously for carcinoma of the lung but was thought to have made a full recovery. Basic life support was performed by the patient's work colleagues and when the paramedics arrived the patient was asystolic. He was treated according to Advanced Life Support (ALS) guidelines ${ }^{1}$ and briefly regained a cardiac output before he arrested again, this time in electro-mechanical dissociation (EMD). A total of $8 \mathrm{mg}$ of adrenaline (epinephrine) was given by the paramedics. On arrival at the accident and emergency department the patient was intubated and cannulated. The endotracheal tube position was checked and breath sounds were audible on both sides of the chest. There was little movement of the left side of the chest and the trachea was deviated towards that side; there were scars consistent with his previous surgery over the left chest wall. The right side of the chest was hyperexpanded.

The patient was in EMD and the ALS protocol was followed. Intravenous fluids were started and after two further $1 \mathrm{mg}$ doses of adrenaline there was a return of spontaneous circulation. Chest radiography was performed. At this time there was no spontaneous respiration and the highest oxygen saturation recorded by pulse oximetry was $80 \%$. The patient briefly arrested again (EMD) but responded to a single $1 \mathrm{mg}$ bolus of adrenaline.

The chest radiograph showed a right sided pneumothorax (fig 1). A 14G intravenous cannula was inserted into the right second intercostal space in the mid-clavicular line and a clear "hiss" of air was heard as the needle was

\section{Accident and \\ Emergency \\ Department, Queen \\ Alexandra Hospital, \\ Southwick Hill Road, \\ Cosham, Portsmouth, Hampshire PO6 3LY, UK}

Correspondence to: Dr Watts

(beverleywatts@ukgateway.net)

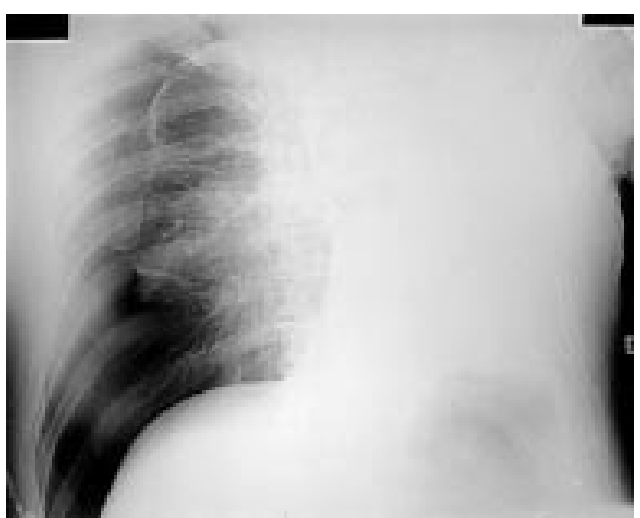

Figure 1 Chest radiograph showing a right sided pneumothorax and mediastinum shifted towards the left. There is a "white out" of the left lung field. withdrawn. The patient's clinical condition immediately improved with oxygen saturation reading $99 \%$ on oxygen via the endotracheal tube. An intercostal tube was inserted (fig 2) and the patient was transferred to the intensive care unit where he unfortunately died approximately 30 hours after admission. A postmortem examination revealed that the cause of the right pneumothorax was a ruptured bulla; the cause of death was cerebral hypoxic damage.

A tension pneumothorax occurs when a one way valve is created between the lung and the pleura. ${ }^{2}$ This leads to an accumulation of air within the pleural cavity during each respiratory cycle, with a consequent increase in intrathoracic pressure. Eventually the ipsilateral lung collapses and the mediastinum is displaced away from the affected side. Venous return is impeded and cardiac arrest ensues. ALS teaches us to consider tension pneumothorax in patients who are in EMD cardiac arrest and to seek the classic findings of a deviated trachea and reduced breath sounds in such patients. In our patient the trachea was deviated to the left and the right side of the chest was hyperexpanded but these clinical findings were anticipated and thought to be secondary to his pneumonectomy. Breath sounds were audible bilaterally, presumably because of some residual lung tissue on the left side. It was not until the chest radiograph became available that the diagnosis of a right pneumothorax was realised. Although the deviated trachea and mediastinal shift were again probably secondary to pneumonectomy (these features remained on the postintercostal drain), the patient's clinical condition suggested that the pneumothorax was under tension and this was confirmed by needle thoracostomy. This is clearly a very unusual situation but serves to remind us that previous pneumonectomy does not preclude a

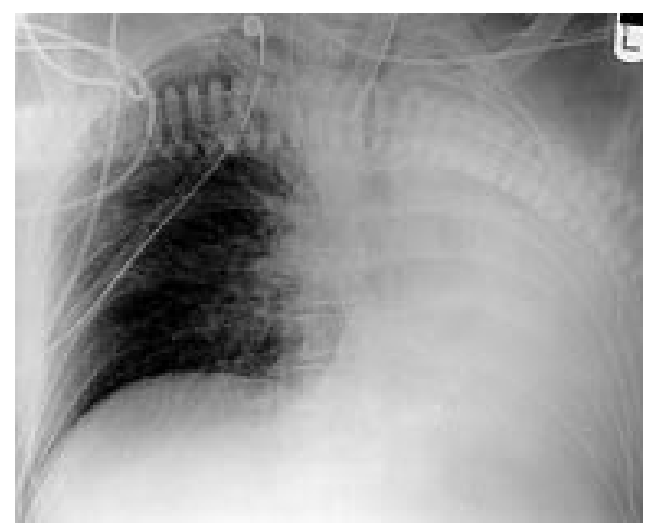

Figure 2 Chest radiograph after intercostal drain insertion showing the reinflated right lung. The mediastinum remains shifted towards the left. 
tension pneumothorax on the opposite side of the chest. Finally, if such a diagnosis is considered, the correct course of action is immediate decompression before obtaining radiological confirmation.
1 Resuscitation Council (UK). Advanced life support manual. 3rd ed. London: 1998.

2 Driscoll PA, Gwinutt CL, Graham TR. Chest and cardiac trauma. In: Skinner D, Swain A, Peyton R, et al, eds. Cambridge textbook of accident and ems. Cambridge: Cambridge University Press, 1997:538.

\title{
An unusual cause of hip pain in a child
}

\author{
M Hynes, F Lam
}

Department of Trauma and Orthopaedics, Chase Farm Hospital, Enfield, Middlesex

Correspondence to: Mr Lam, 4 Middlefield, St John's Wood, London NW8 6NE

(mrlam@hotmail.com)
An 11 year old boy presented to the accident and emergency department complaining of left hip pain after a fall from his skateboard. Apart from a mild restriction of hip movement caused by pain, physical examination and a pelvic radiograph in the AP view were unremarkable. He was discharged home with simple analgesia.

He presented again two weeks later with worsening pain and a limp. Examination revealed exquisite tenderness in his gluteal muscles with a markedly reduced range of movement. Repeat pelvic radiographs including $\mathrm{AP}$ and frog lateral views were performed showing a calcified mass in the abductor mechanism of his left hip (fig 1). In view of the unresolving pain, magnetic resonance imaging was performed showing high signalling within the mass with distortion of the adjacent muscular structure associated with marked soft tissue swelling and an effusion in the hip joint (fig 2). This raised the suspicion of a tumour. Consequently, a computed tomography guided biopsy was performed showing histological features of reactive new bone formation, suggestive of myositis ossificans. ${ }^{1}$ The patient was subsequently treated with indomethacin and symptoms resolved a few weeks later.

Myositis ossificans is the formation of mature bone outside the skeleton. Although it is an entirely benign condition, it is nevertheless an important condition because in its early stages presentation may be difficult to distinguish both clinically and radiologically from a soft tissue tumour such as rhabdomyosarcoma, which is the commonest soft tissue

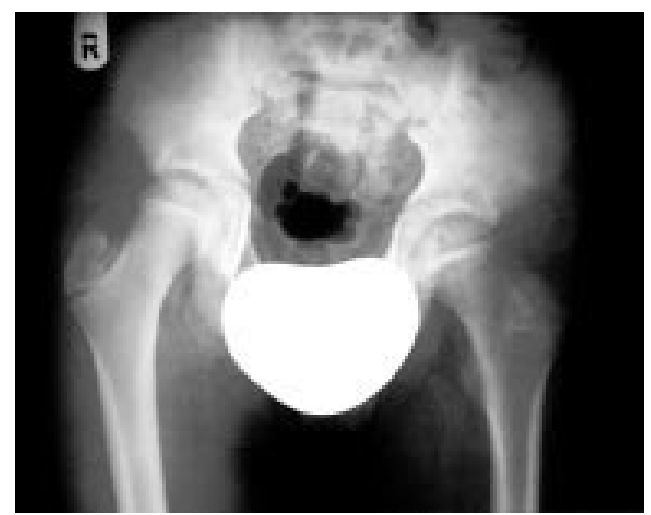

Figure 1 Calcified mass in the abductor mechanism of the left hip.

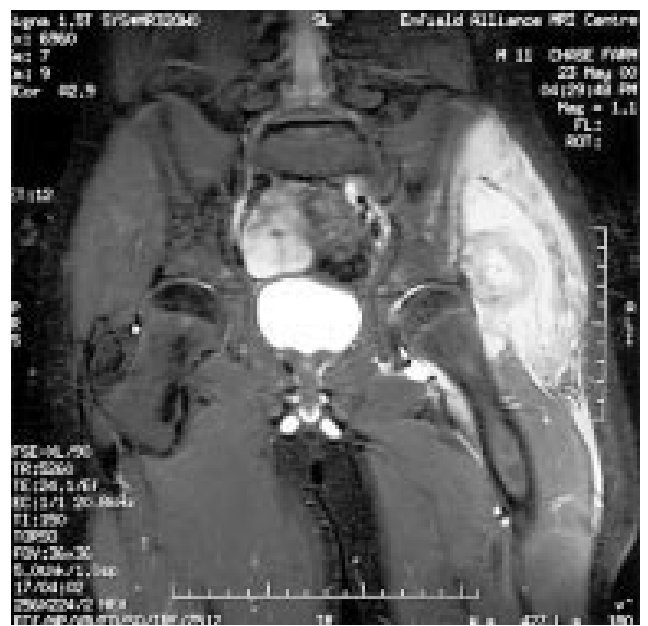

Figure 2 Abnormal high signalling within the mass with marked surrounding soft tissue swelling.

ferential diagnoses would include a calcifying haematoma, slipped capital femoral epiphysis and septic arthritis. The other important learning point is that any child with a persisting limp should be evaluated radiologically with two views including a frog lateral because conditions such as slipped capital femoral epiphysis are easily missed on the AP view alone.
1 Hanquinet S, Ngo L, Anooshiravani M, et al. Magnetic resonance imaging helps in the early diagnosis of myositis ossificans in children. Pediatric Surgery International ossificans in $1999 ; 15: 287-9$.

2 de Almeida MM, Abecassis N, Almeida MO, et al. Fine needle aspiration cytology of myositis ossificans: a case report. Diagn Cytopathol 1994;10:41-3. 